\title{
Indicaciones de trasplante pulmonar según patología de base
}

\author{
MARÍA T. PARADA C.* y CLAUDIA SEPÚLVEDA L.**
}

\section{Selection of lung transplant candidates according the underlying disease}

The presence of a respiratory disease at an advanced course causes a poor quality of life and a low survival, so that lung transplantation is an option for a group of patients. The progression of the disease is variable and specific criteria should be considered for selecting transplant candidates according the underlying disease. The decision to enter a patient into a waiting list is complex and involves an addition of several clinical variables, psychological and social assessments, so referring a patient to a transplant center not always makes sure the possibility of reaching a transplant. The timing of referral and the admission to listing are different and it is important to have time for analysis, because of the limited supply of donors. The process for selecting a candidate must assess the condition of underlying disease and the likelihood of surviving the transplant procedure.

Key words: Lung transplantation, interstitial lung diseases, cystic fibrosis, pulmonary hypertension, emphysema.

\section{Resumen}

La presencia de una patología respiratoria avanzada provoca una deficiente calidad de vida y escasa sobrevida, por lo que el trasplante pulmonar es una opción para un grupo de pacientes. La progresión de la enfermedad es variable y existen diferentes criterios a considerar para la selección de candidatos a trasplante según la patología de base. La decisión de ingresar a lista de espera es compleja e involucra además de variables clínicas de la enfermedad, las evaluaciones psicológicas y sociales, por lo que la derivación a un centro no constituye siempre la posibilidad de alcanzar un trasplante. El momento de la derivación y el momento del ingreso a la lista son diferentes y es importante contar con un tiempo de análisis, ante la escasa oferta de donantes. El proceso de selección de un candidato debe evaluar las condiciones de la patología de base y la probabilidad de sobrevivir al procedimiento del trasplante.

Palabras clave: Trasplante pulmonar, enfermedad intersticial pulmonar, fibrosis quística, hipertensión pulmonar, enfisema.

\section{Introducción}

El trasplante pulmonar es una técnica ofrecida a aquellos pacientes portadores de patología pulmonar avanzada y refractaria a tratamiento médico quienes deben cumplir con los siguientes criterios generales:

- Alto riesgo de morir por la patología pulmonar en los próximos dos años.

- Alta probabilidad de sobrevivir en los próximos 90 días del trasplante.

- Alta probabilidad de sobrevivir a los 5 años.
La apropiada selección de los receptores es importante para determinar los resultados posteriores.

La Sociedad Internacional de Trasplante Corazón-Pulmón (ISHLT), ha desarrollado guías de selección de candidatos por patología, publicadas en 1998-2006 ${ }^{1}$ y actualizadas en el año $2015^{2}$ cuyo fin es asistir a médicos especialistas para referir a un centro de trasplante y a aquellos que trabajan en esta área, poder identificar los pacientes que pueden lograr mayor beneficio de este procedimiento.

\footnotetext{
* Centro de Trasplantes Clínica Las Condes. Las Condes. RM. Chile.

** Instituto Nacional del Tórax. Providencia, RM. Chile.
} 
La escasez de donantes es la limitación primaria al trasplante por lo que los criterios de selección son estrictos y debe identificarse la forma de progresión de la enfermedad, para disminuir el riesgo de fallecer en lista de espera. En Estados Unidos desde el año 2005 se aplica un criterio de puntuación de gravedad denominado LAS (Lung Allocation Score) que ha permitido facilitar el trasplante a pacientes portadores de Fibrosis pulmonar y ha disminuido su frecuencia para pacientes con $\mathrm{EPOC}^{3}$.

El manejo de las nuevas terapias en Hipertensión pulmonar ha llevado a una disminución significativa de la necesidad de trasplante pulmonar y el trasplante cardiopulmonar constituye una excepción en la actualidad.

El conocimiento de los criterios de derivación según patología es importante para los especialistas en enfermedades respiratorias.

\section{Proceso de selección}

Consta de dos etapas definidas:

- Estudio del candidato: Se analiza extensamente si existe compromiso de otros órganos que pueda contraindicar el procedimiento, o si existen patologías que deban corregirse previo al trasplante.

- Paciente incluido en la lista: Se realiza educación sobre oxigenoterapia, rehabilitación, alimentación, administración de vacunas, soporte psicológico y psiquiátrico y control seriado de función pulmonar para evaluar el grado de deterioro ante eventual necesidad de urgencia.

En este análisis debe buscarse las condiciones que no permitan a un paciente el postular al proceso de un trasplante pulmonar.

- Contraindicaciones absolutas: Se describen en Tabla 1 y es de especial consideración evaluar la adherencia al tratamiento de los pacientes y el soporte social que presentan.

- Contraindicaciones relativas: Se consideran aquellas que pueden presentar corrección y luego de una posterior reevaluación permitan la aceptación del candidato:

- Edad > 65 años (es evaluable de acuerdo a protocolo de cada centro).

- Infección crónica por Hepatitis B-C (sin cirrosis hepática o hipertensión portal).

- Pobre potencial de rehabilitación.

- Colonización por gérmenes altamente resistentes: bacterias, hongos y mycobacterias.

- IMC (índice de masa corporal) $>30 \mathrm{o}<17$ $\mathrm{kg} / \mathrm{m}^{2}$.
- Osteoporosis severa sintomática.

- Cirugía torácica previa con pleurodesis por riesgo de sangramiento.

- Condiciones médicas no tratadas: Reflujo gastroesofágico (RGE) severo, úlcera péptica, enfermedad coronaria no corregida.

\section{Selección de candidatos por patología}

El momento de la indicación es variable de acuerdo a la patología de base del paciente. Existen criterios de derivación a un centro de trasplante y criterios propios para ingresar a lista de espera.

El enlistamiento de un paciente ocurre cuando la esperanza de vida sin trasplante es menor al riesgo de ser sometido a este procedimiento $\mathrm{y}$ existe refractariedad a tratamiento médico.

Ante la escasez de donantes, el momento de derivación no debe tardar porque disminuye la posibilidad de contar con el tiempo adecuado para alcanzar el trasplante.

\section{Enfermedad pulmonar intersticial}

Los pacientes portadores de fibrosis pulmonar idiopática tienen el peor pronóstico entre las indicaciones de trasplante pulmonar alcanzando una sobrevida de 20 a $30 \%$ a 5 años una vez hecho el diagnóstico ${ }^{4}$. Otro tipo de enfermedades intersticiales si alcanzan refractariedad al tratamiento tienen también un pronóstico ominoso. La enfermedad pulmonar asociada a colagenopatías ocupa criterios similares a la enfermedad idiopática ${ }^{7}$.

Tabla 1. Contraindicaciones absolutas de trasplante pulmonar

- Enfermedad neoplásica en los últimos 2 años. Sin embargo, se sugiere un intervalo $>5$ años libre de enfermedad; excepto las neoplasias cutáneas no melanoma

- Disfunción avanzada e intratable de otro órgano (corazón, hígado y riñón)

- Condición clínica inestable (enfermedad coronaria aguda, sepsis)

- Deformidad importante de la pared torácica

- Infección por gérmenes de difícil tratamiento

- No adherencia o incapacidad de seguir tratamiento y controles

- Condición psiquiátrica o psicológica intratable

- Ausencia de soporte social y familiar

- Adicción activa a sustancias: alcohol, tabaco y drogas, en los últimos 6 meses 
Tabla 2. Indicaciones de trasplante pulmonar por patología

\begin{tabular}{|c|c|c|}
\hline Patología & Criterios de derivación & Criterios de trasplante \\
\hline Fibrosis pulmonar & $\begin{array}{l}\text { - Evidencia histológica o radiológica de } \\
\text { UIP o NSIP fibrosa }\end{array}$ & $\begin{array}{l}\text { - DLco }<39 \% \\
\text { - Caída de CVF }>10 \% \text { en } 6 \text { meses } \\
\text { - Desaturación }>88 \% \text { en TC6min } \\
\text { - Panal en TAC con puntaje de fibrosis }>2\end{array}$ \\
\hline $\begin{array}{l}\text { Fibrosis quística }(\mathrm{FQ}) \\
\text { Otras bronquiectasias no FQ }\end{array}$ & $\begin{array}{l}\text { - } \mathrm{VEF}_{1}<30 \% \text { o rápido deterioro } \\
\text { - Exacerbación que requiere UCI } \\
\text { - Aumento frecuencia exacerbaciones que } \\
\text { requieren antibióticos } \\
\text { - Neumotórax refractario y/o recurrente } \\
\text { - Hemoptisis recurrente no controlada con } \\
\text { embolización }\end{array}$ & $\begin{array}{l}\text { - Oxigeno dependiente } \\
\text { - Hipercapnia } \\
\text { - Hipertensión arterial pulmonar (HTP) }\end{array}$ \\
\hline EPOC & - $\mathrm{BODE}>5$ & $\begin{array}{l}\text { - } \mathrm{BODE}>7 \\
\text { - Hospitalización con hipercapnia } \\
(>50 \mathrm{mmHg}) \\
\text { - } \mathrm{HTP} \text { a cor pulmonale } \\
\text { - } \mathrm{VEF}_{1}<20 \% \text {, DLco }>20 \% \text { con enfise- } \\
\text { ma homogéneo }\end{array}$ \\
\hline $\begin{array}{l}\text { Hipertensión arterial } \\
\text { Pulmonar (HTP) }\end{array}$ & $\begin{array}{l}\text { - CF III-IV con terapia médica máxima } \\
\text { - Enfermedad rápidamente progresiva }\end{array}$ & $\begin{array}{l}\text { - TC6min }<350 \mathrm{~m} \\
\text { - Falla con terapia epoprosterenol o } \\
\text { equivalente } \\
\text { - } \mathrm{IC}<2 \mathrm{~L} / \mathrm{min} / \mathrm{m}^{2} \\
\text { - Presión aurícula derecha }>15 \mathrm{mmHg}\end{array}$ \\
\hline
\end{tabular}

UIP: Usual Interstitial Pneumonia; NSIP: Non Specific Interstitial Pneumonia; TAC: tomografía axial computarizada; DLCO: Capacidad de difusión con CO; TC6min: Test de caminata de 6 min; IC: índice cardiaco; CF: Capacidad funcional (ver texto).

\section{Indicación de referencia}

- Capacidad vital forzada (CVF) $<80 \%$ o capacidad de difusión de CO (DLco) $<40 \%$.

- Requerimiento de $\mathrm{O}_{2}$ aunque sólo sea en test de caminata de 6 minutos (TC $6 \mathrm{~min}$ ).

- Histología de UIP (usual interstitial pneumonia).

- Falta de respuesta a tratamiento médico.

\section{Indicación de ingreso a lista de espera}

- Caída de CVF $>10 \%$ en 6 meses.

- Caída de DLco $>15 \%$ en 6 meses.

- TC6 min $<250$ m o caída de la $\mathrm{SpO}_{2}<88 \%$.

- Hipertensión arterial pulmonar.

- Historia de exacerbación.

\section{Fibrosis quística (FQ)}

Si bien es una enfermedad con compromiso de varios órganos, el $85 \%$ de los pacientes que fallecen lo hacen por una complicación pulmonar ${ }^{5}$. Este grupo de pacientes una vez que adquieren gérmenes de mayor resistencia o cursan con exacerbaciones frecuentes presentan un deterioro progresivo de su función pulmonar y de su estado general.

En el registro de ISHLT los portadores de FQ presentan la mejor sobrevida promedio post trasplante la cual llega a 8,3 años $^{6}$.

\section{Indicación de referencia}

- Volumen espiratorio forzado en el $1^{\mathrm{er}} \mathrm{s}\left(\mathrm{VEF}_{1}\right)$ $<30 \%$ o deterioro rápidamente progresivo especialmente en mujeres a pesar de óptimo tratamiento médico.

- Exacerbación que requiere de manejo en Unidad de Cuidados Intensivos.

- Incremento de exacerbaciones y aumento de resistencia bacteriana.

- Hipertensión arterial pulmonar no asociada a hipoxemia.

- Neumotórax recurrente.

- Hemoptisis recurrente.

\section{Indicación de ingreso a lista de espera}

- Insuficiencia respiratoria.

- Hipercapnia.

- Necesidad de ventilación mecánica no invasiva.

- Hipertensión arterial pulmonar.

\section{Consideraciones especiales}

Todos los pacientes portadores de FQ derivados a un centro de trasplantes deben ser evaluados para Mycobacteria no TBC, de encontrarse $(+)$ debe realizar tratamiento previo a ingreso a la lista. La falta de respuesta a terapia o el compromiso extrapulmonar contraindican el trasplante. Así mismo debe buscarse en forma dirigida la 
presencia de Burkholderia cepacia complex $(B c c)$, quienes se deterioran en forma acelerada y la presencia del tipo B cenocepacia constituye una contraindicación absoluta al procedimiento por la alta mortalidad peri-operatoria y mal resultado a largo plazo $^{7-8}$.

\section{Enfermedad pulmonar obstructiva crónica (EPOC)}

El $40 \%$ de los trasplantes pulmonares el año 2014, fue por EPOC, sin embargo, desde la implementación del LAS (2005), ha ido en aumento la proporción de trasplantes por fibrosis pulmonar idiopática9.

Claramente la EPOC tiene un curso más lento y mejor sobrevida que otras enfermedades pulmonares, pero con una muy mala calidad de vida.

Lo que ha demostrado ser un buen índice evaluador es el puntaje BODE, que en los puntajes más altos se correlaciona con enfermedad más severa y peor sobrevida. Se ha observado que el deterioro de 1 punto de BODE entre 6 y 24 meses aumenta la mortalidad al doble ${ }^{10}$.

Especial consideración merece mencionar los pacientes con enfisema apical, que debieran ser evaluados primariamente para una cirugía de reducción de volumen.

\section{Indicaciones de referencia}

- BODE $>5$.

\section{Indicaciones de trasplante}

- BODE $>7$.

- Hospitalización con hipercapnia (> $50 \mathrm{mmHg}$ ).

- Hipertensión arterial pulmonar o cor pulmonale.

- $\mathrm{VEF}_{1}<20 \%$, DLco $>20 \%$ con enfisema homogéneo.

\section{Hipertensión arterial pulmonar primaria (HTP)}

El desarrollo de nuevas terapias médicas ha retrasado la indicación y enlistamiento de los pacientes por hipertensión arterial pulmonar (HTP). Se han identificado factores que aumentan la mortalidad: Clase IV de la NYHA, sexo masculino, $>60$ años, aumento de la resistencia vascular pulmonar, hipertensión portal, historia familiar de HTP, aumento del pro-BNP (Brain natriuretic peptide), insuficiencia renal, HTP secundaria a colagenopatías y derrame pericárdico. En este grupo de pacientes, a pesar de la terapia médica, deben ser referidos precozmente a un centro de trasplante.

\section{Indicaciones de referencia}

- Capacidad funcional III-IV con terapia médica máxima.

- Enfermedad rápidamente progresiva.

\section{Indicaciones de trasplante}

- TC6M $<350 \mathrm{~m}$.

- Falla con terapia epoprosterenol o equivalente.

- Índice cardíaco (IC) $<2 \mathrm{~L} / \mathrm{min} / \mathrm{m}^{2}$.

- Presión de aurícula derecha $>15 \mathrm{mmHg}$.

\section{Consideraciones especiales en indicación de trasplante pulmonar}

\section{Retrasplante}

Constituye un escaso número de trasplantes, sin embargo, ha aumentado en los últimos años en Estados Unidos y es de elección para pacientes con disfunción crónica de injerto de tipo síndrome de bronquiolitis obliterante (SBO) desarrollado después de dos años del trasplante inicial ${ }^{11}$. La opción para pacientes con disfunción primaria de injerto ha dado muy malos resultados.

\section{Trasplante corazón-pulmón}

Es una opción considerada principalmente para pacientes portadores de cardiopatía congénita no corregida con hipertensión arterial pulmonar secundaria severa ${ }^{12}$. En cambio los pacientes portadores de hipertensión arterial pulmonar primaria asociada a falla ventricular derecha se benefician de un trasplante bipulmonar con buenos resultados ${ }^{13}$.

\section{Disfunción de esófago en esclerodermia}

Los resultados permanecen controversiales en esclerodermia por la asociación de falta de motilidad esofágica y gastroparesia que favorece el desarrollo de SBO precoz asociado al reflujo gastroesofágico. La cuidadosa selección de estos pacientes y el buen manejo digestivo pueden lograr resultados satisfactorios a largo plazo ${ }^{14}$.

\section{Conclusiones}

El diferente compromiso de función pulmonar por patología ha llevado al desarrollo de guías de selección acuciosa de candidatos a trasplante. Destaca el momento de derivación a un centro especializado, la búsqueda de contraindicaciones y finalmente a la elección de los mejores candidatos a recibir un órgano y lograr la mejor calidad de vida y sobrevida a largo plazo. 


\section{Bibliografía}

1.- INTERNATIONAL GUIDELINES FOR THE SELECTION OF LUNG TRANSPLANT CANDIDATES: 2006 Update-A Consensus Report From the Pulmonary Scientific Council of the International Society for Heart and Lung Transplantation. J Heart Lung Transplant 2006; 25: 745-55.

2.- WEILL D, BENDEN C, CORRIS P A, DARK JH, DAVIS R D, KESHAVJEE S, et al. A consensus document for the selection of lung transplant candidates. J Heart Lung Transplant 2015; 34: 1-15.

3.- GRIES C J, RUE T C, HEAGERTY P J, EDELMAN J D, MULLIGAN M S, GOSS C H, et al. Development of a predictive model for long-term survival after lung transplantation and implications for the lung allocation score. J Heart Lung Transplant 2010; 29: 731-8.

4.- KISTLER K D, NALYSNYK L, ROTELLA P, ESSER D. Lung transplantation in idiopathic pulmonary fibrosis. A systematic review of the literature. BMC Pulm Med 2014; 14: 139 doi: 10.1186/1471-2466-14-139. Review.

5.- CORRIS P. Lung Transplantation for Cystic Fibrosis and Bronchiectasis. Semin Respir Crit Care Med 2013; 34: 297-304.

6.- THE REGISTRY OF THE INTERNATIONAL SOCIETY FOR HEART AND LUNG TRANSPLANTATION: Thirty-first Adult Lung and Heart-Lung Transplant Report-2014; Focus Theme Retransplantation. J Heart Lung Transplant 2014; 33: 1009-24.

7.- DE SOYZA A, CORRIS P A. Lung transplantation and the Burkholderia cepacia complex. J Heart Lung Transplant 2003; 22: 954-8.
8.- DE SOYZA A, MEACHERY G, HESTER K, NICHOLSON A, PARRY G, TOCEWICZ K, et al. Lung transplantation for patients with cystic fibrosis and Burkholderia cepacia complex infection: a single center experience. J Heart Lung Transplant 2010; 29: 1395 404.

9.- MCCURRY K R, SHEARON T H, EDWARDS L B, CHAN K M, SWEET S C, VALAPOUR M, et al. Lung transplantation in the United States, 1998-2007. Am J Transplant 2009; 9 (4 Pt 2): 942-58.

10.- MARTÍNEZ F J, HAN M K, ANDREI A C, WISE R, MURRAY S, CURTIS J L, et al. National Emphysema Treatment Trial Research Group. Longitudinal change in the BODE index predicts mortality in severe emphysema. Am J Respir Crit Care Med 2008; 178: 491-9.

11.- KAWUT S M. Lung retransplantation. Clin Chest Med 2011; 32: 367-77.

12.- CHOONG C K, SWEET S C, GUTHRIE T J, MENDELOFF E N, HADDAD F J, SCHULER P, et al. Repair of congenital heart lesions combined with lung transplantation for the treatment of severe pulmonary hypertension: a 13-year experience. J Thorac Cardiovasc Surg 2005; 129: 661-9.

13.- FADEL E, MERCIER O, MUSSOT S, LEROY-LADURIE F, CERRINA J, CHAPELIER A, et al. Long-term outcome of double-lung and heart-lung transplantation for pulmonary hypertension: a comparative retrospective study of 219 patients. Eur J Cardiothorac Surg 2010; 38: 277-84.

14.- SOTTILE P D, ITURBE D, KATSUMOTO T R, et al. Outcomes in systemic sclerosis-related lung disease after lung transplantation. Transplantation 2013; 95: 975-80.

Correspondencia a:

Dra. María T. Parada C.

Centro de Trasplantes Clínica Las Condes

Lo Fontecilla 441 Las Condes. RM. Chile.

Email: mtparada@clc.cl 